\title{
Development of a high-density highly oriented graphite stripper
}

\author{
Hiroo Hasebe ${ }^{1, *}$ Hiroki Okuno ${ }^{1}$, Atsushi Tatami ${ }^{2}$, Masamitsu Tachibana ${ }^{2}$, Mutsuaki Murakami ${ }^{2}$, Hiroshi Imao $^{1}$, \\ Nobuhisa Fukunishi ${ }^{1}$, Masayuki Kase ${ }^{1}$, and Osamu Kamigaito ${ }^{1}$ \\ ${ }^{1}$ Nishina Center for Accelerator-Based Science, RIKEN, 2-1 Hirosawa, Wako, Saitama 351-0198, Japan \\ ${ }^{2}$ Material Solutions New Research Engine, Kaneka Corporation, 5-1-1 Torikainishi, Settsu, Osaka 566-0072, Japan
}

\begin{abstract}
High-density highly oriented graphite sheets provided by Kaneka Corporation have been applied as stripper disks for heavy-ion acceleration at RIKEN RI Beam Factory since 2014. The graphite sheets withstand increasing amounts of beam intensity. We observed the graphite sheets after beam irradiation with an electron microscope and their lifetime was discussed.
\end{abstract}

\section{Introduction}

Among many heavy-ion accelerator facilities, static-type carbon foils (C-foils) have been used as charge strippers in heavy ion acceleration for a long time. In recent years, many accelerator facilities have upgraded or plan to upgrade with the aim of providing a higher beam intensity and higher beam energy. Inevitably, the lifetime of the charge stripper foil has become a serious concern for the accomplishment of beam operations. This has led to the replacement of foil strippers, such as carbon and thin metal foils, with gases with low atomic numbers, like $\mathrm{He}$ and $\mathrm{H}_{2}$, or flowing liquid metals.

For uranium (U) acceleration at the RIKEN RI Beam Factory (RIBF), the charge state of $\mathrm{U}^{86+}$ is necessary to accelerate $U$ ions up to the final energy of $345 \mathrm{MeV} /$ nucleon. It is difficult to obtain such a high charge state at the stripping energy around $50 \mathrm{MeV} /$ nucleon without C-foil strippers. Before 2011, we used static-type polycrystalline graphite foils with a thickness of $17 \mathrm{mg} \mathrm{cm}^{-2}$ fabricated by Arizona Carbon Foil Company [1]. Beryllium disks [2] (with a thickness of $17 \mathrm{mg} \mathrm{cm}^{-2}$ ) were used from 2012 to 2014. In 2014, we found that high-density highly oriented graphite sheets (GSs), provided by Kaneka Corporation [2, 3], can be applied as the stripper disks for heavy ion acceleration. GS-disks have been used since 2015.

\section{Graphite sheet disk stripper}

\subsection{Characteristics of the GSs}

The GSs are prepared from heat-treated polyimide films at temperatures up to $3000^{\circ} \mathrm{C}$ [3]. The characteristics of the GSs are listed in Table 1. A prominent feature of the GSs is their very high thermal conductivity of $1500 \mathrm{~W} \mathrm{~m}^{-1} \mathrm{~K}^{-1}$ in the plane direction. In addition, the
GSs have a higher thermal diffusivity than copper or aluminum. The GSs have a high density and uniform thickness.

Table 1. Characteristics of the KANEKA GS.

\begin{tabular}{|l|l|l|l|}
\hline \multicolumn{2}{|l|}{} & Units & $\begin{array}{l}\text { Typical } \\
\text { values }\end{array}$ \\
\hline \multirow{2}{*}{$\begin{array}{l}\text { Thermal } \\
\text { conductivity }\end{array}$} & $\begin{array}{l}\text { In plane } \\
\text { (XY axis) }\end{array}$ & \multirow{2}{*}{$\begin{array}{l}\text { Normal to } \\
\text { plane (Z axis) }\end{array}$} & $\mathbf{1 5 0 0}$ \\
\cline { 2 - 4 } & & 5 \\
\cline { 3 - 4 } & $\mathrm{cm}^{2} \mathrm{~s}^{-1}$ & 9.0 \\
\hline Thermal diffusivity & $\mathrm{g} \mathrm{cm}^{-3}$ & $\mathbf{2 . 0}$ \\
\hline Density & $\mathrm{MPa}$ & 40 \\
\hline \multicolumn{2}{|l|}{ Tensile strength } & $\mathrm{Cycles}$ & $>10000$ \\
\hline Bending & $\mathrm{S} \mathrm{cm}^{-1}$ & 13000 \\
\hline Electrical conductivity & & \\
\hline
\end{tabular}

\subsection{GS-disk used in U beam time}

The GSs are mechanically strong and can be shaped easily by scissors or a cutter knife. Since the thickness variation of the GS sample was limited, we prepared a new GS-disk by overlapping two sheets so the total thickness became $15 \mathrm{mg} \mathrm{cm}^{-2}$, which is necessary for $\mathrm{U}$ beam acceleration. Each sheet, with a thickness of $7.5 \mathrm{mg} \mathrm{cm}^{-2}$, was cut to a circular shape with an outer diameter of $110 \mathrm{~mm}$.

Figure 1 shows a photograph of the four GS-disks after $\mathrm{U}$ beam irradiation during the operation period from 2015 to 2017 . We do not observe any serious damage by beam irradiation on any disk. Only slight deformation and beam irradiation marks are observed. Disks 1 and 2 were used for two beam times. The beam intensity after the stripper fluctuated slightly at the end of the beam time. Disks 3 and 4 provided an excellent beam during the beam time with no damage and no beam intensity fluctuation, however their use was discontinued after one beam time.

\footnotetext{
* Corresponding author: hasebe@riken.jp
} 

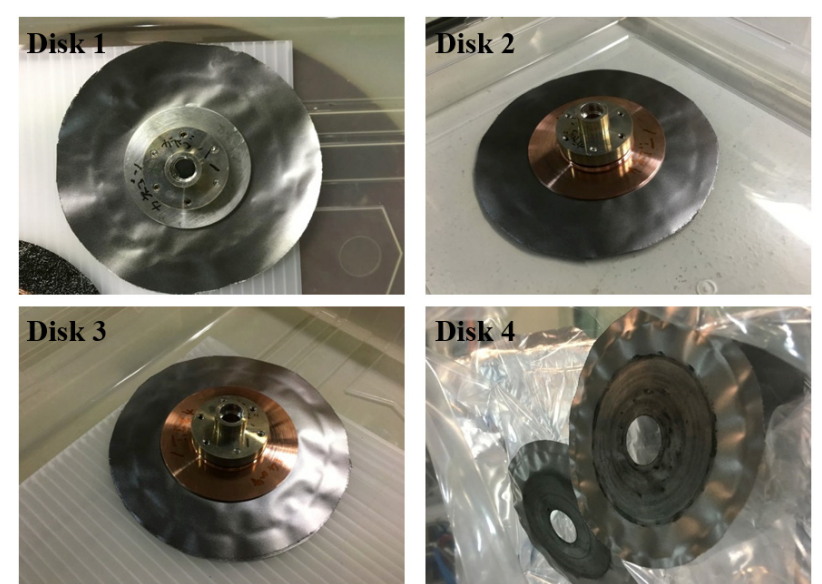

Fig. 1. Four GS-disks after the beam times.

Table 2 summarizes the results of the four GS-disks. The highest beam intensity of $20 \mathrm{e} \mu \mathrm{A}$ was irradiated on Disk 4. However, the intensity downstream did not fluctuate. The total beam particles irradiated on Disks 1 and 2 are shown in Table 2. For Disks 3 and 4, estimations of the irradiated beam amounts are shown. The disks were only slightly deformed. Their actual lifetimes are longer than the shown values. They may be able to cope with a further increase in beam intensity, however, introducing new measures to suppress deformation is necessary in future.

\section{Observation scanning electron microscopy}

Disk 1 was observed with a scanning electron microscope (SEM) with the electronic scanning micro analyzer (EPMA). A part of the beam irradiation mark was cut with scissors and was placed at the sample stage of the SEM. The surface, the edge surface, and the crosssection were observed in both cases in which the beam was irradiated or not irradiated. To observe the crosssection, a GS was attached to a brass block and was placed upright. As shown in Figure 1, we cannot see any damage by eye to the GS.

\subsection{SEM images of the GS surface}

Figures 2 (a1) and (a2) show the SEM images of the noirradiated part, and (b1) and (b2) show the beamirradiated part. The magnification factors are 1000 times for (a1) and (b1), and 5000 times for (a2) and (b2). In the SEM image with 5000-times magnification, damage of micrometer-order can be seen at the beam irradiated part.
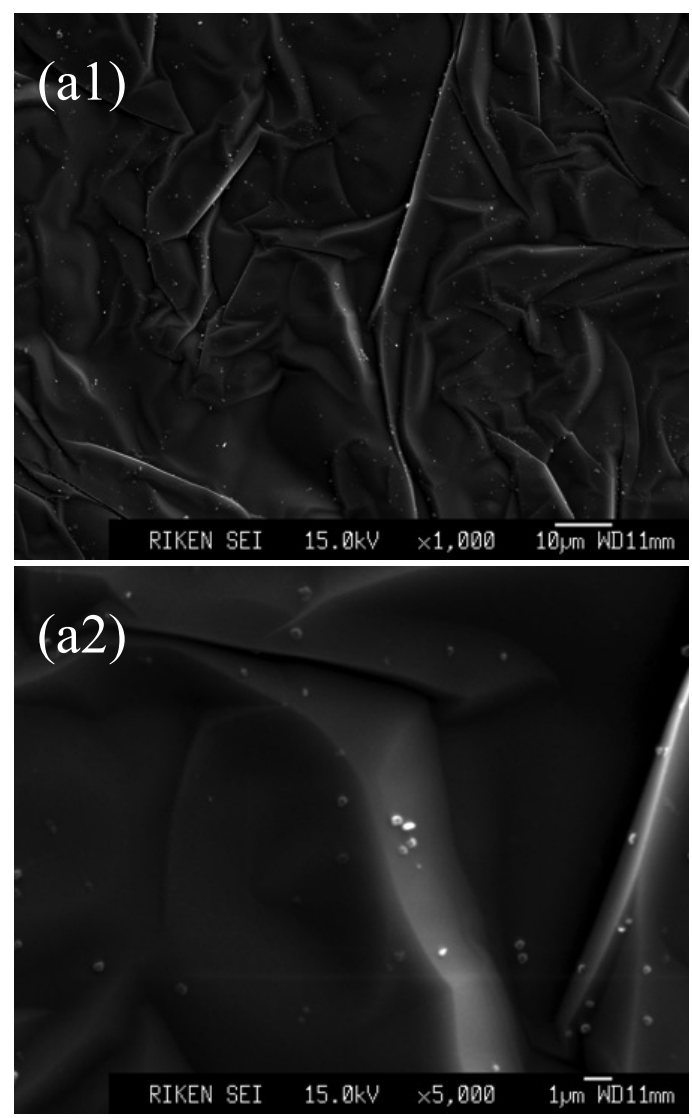

Fig. 2a. SEM images of the GS surface. (a1) and (a2): noirradiated part.

Table 2. Summary of the results of 4 GS-disks.

\begin{tabular}{|c|c|c|c|c|}
\hline Disk name & $\begin{array}{c}\text { Maximum } \\
\text { Beam intensity } \\
(\mathrm{e} \mu \mathrm{A})\end{array}$ & $\begin{array}{c}\text { Total irradiated } \\
\text { particles }\end{array}$ & $\begin{array}{c}\text { Irradiated } \\
\text { period } \\
(\text { Days })\end{array}$ & During irradiation \\
\hline Disk 1 & 17.5 & $2.19 \times 10^{18}$ & 40 & Slight beam fluctuation \\
\hline Disk 2 & 11.5 & $1.6 \times 1^{18}$ & 52 & Slight beam fluctuation \\
\hline Disk 3 & 15.8 & $\begin{array}{c}1.2 \times \mathbf{1 0}^{18} \\
(\text { Estimation })\end{array}$ & 25 & No beam fluctuation \\
\hline Disk 4 & 20 & $\begin{array}{c}2.5 \times 10^{18} \\
(\text { Estimation })\end{array}$ & 43 & $\begin{array}{c}\text { No beam fluctuation } \\
\text { Deformed a little }\end{array}$ \\
\hline
\end{tabular}



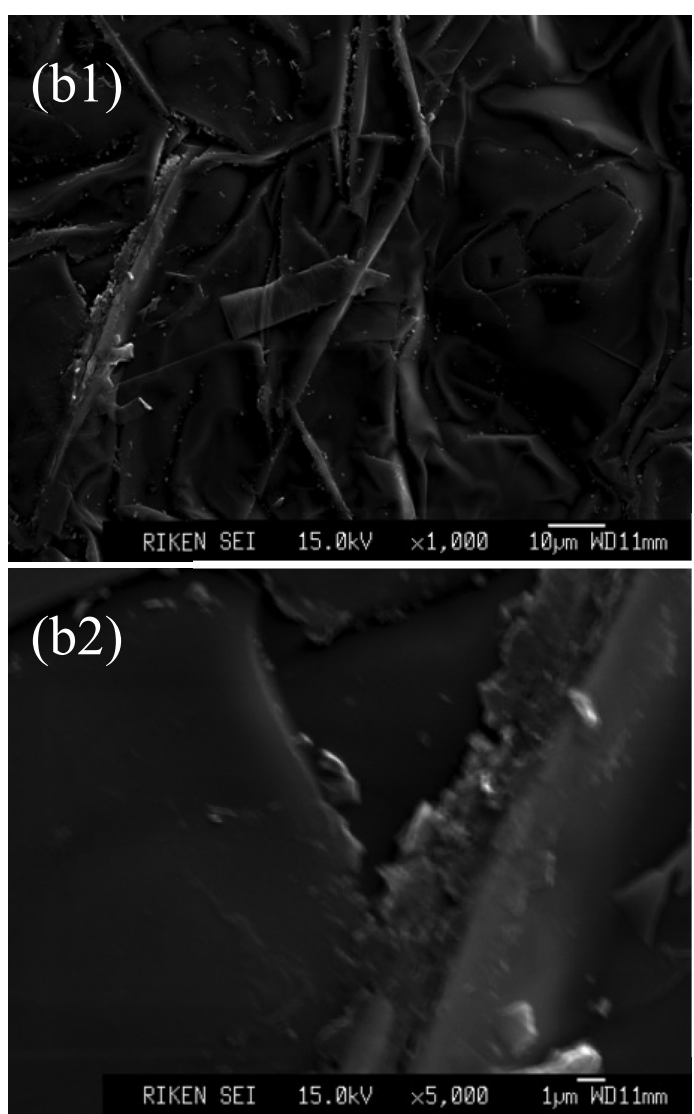

Fig. 2b. SEM images of the GS surface. (b1) and (b2): beamirradiated part.
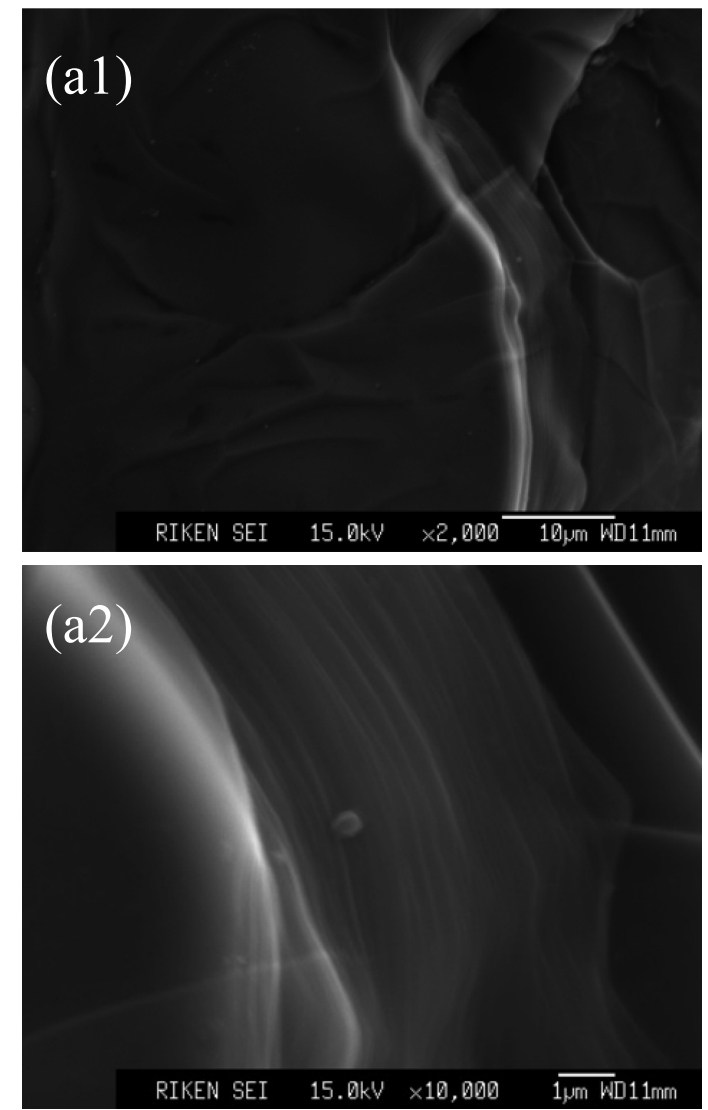

Fig. 3a. SEM images of the GS surface edge taken from above. (a1) and (a2): no-irradiated part.

\subsection{SEM images of the GS surface at the edge from above}

Figures 3 show the SEM images taken of the GS surface edge from above. Figure (a1) and (a2) show the noirradiated part, and (b1) and (b2) show the beamirradiated part. The magnification factors are 2000 times for (a1) and (b1), and 10,000 times for (a2) and (b2). As well as the surface, you can also see damage of micrometer-order at the edge of the beam-irradiated part. A smooth multilayer structure is observed at the noirradiated part.
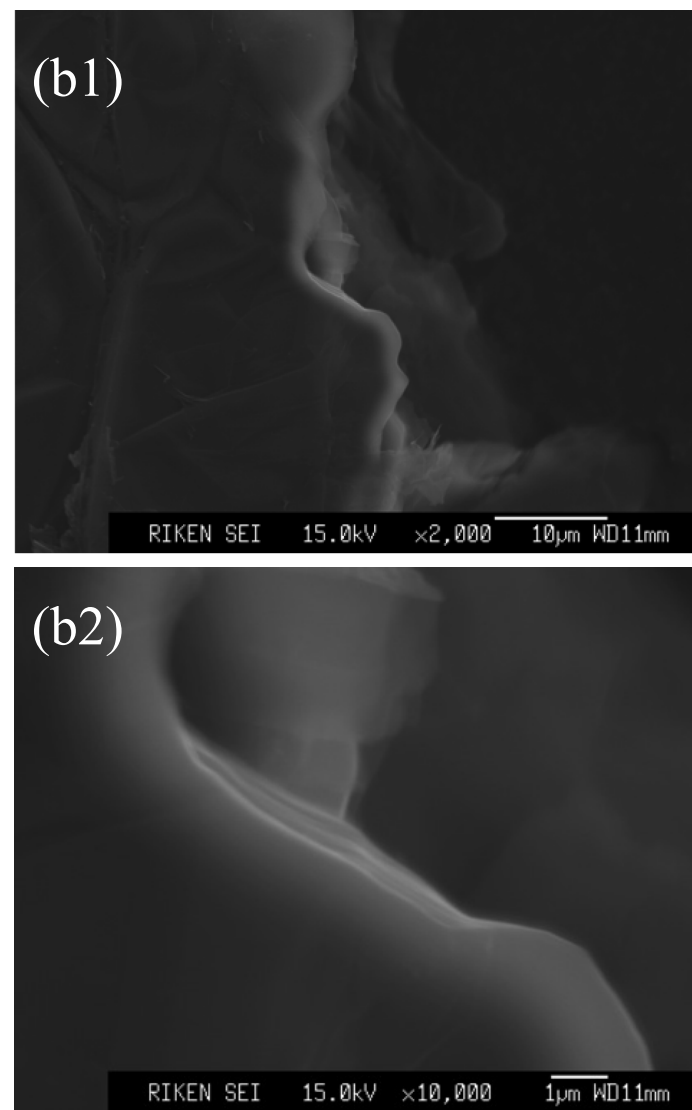

Fig. 3b. SEM images of the GS surface edge taken from above. (b1) and (b2): beam-irradiated part.

\subsection{SEM images of the GS cross-section}

Figures 4 show the SEM images observing the GS crosssection. Figure (a1) and (a2) show the no-irradiated part, and (b1) and (b2) show the beam-irradiated part. The magnification factors are 2500 times for (a1) and (b1), and 10,000 times for (a2) and (b2). You can also see damage of micrometer-order at the center of the irradiated part. However, we cannot observe the change in the thickness. To investigate the carbon purity, EPMA analysis was also performed in both cases for the beam irradiated and noirradiated parts. Substances other than carbon were not detected at any part. 

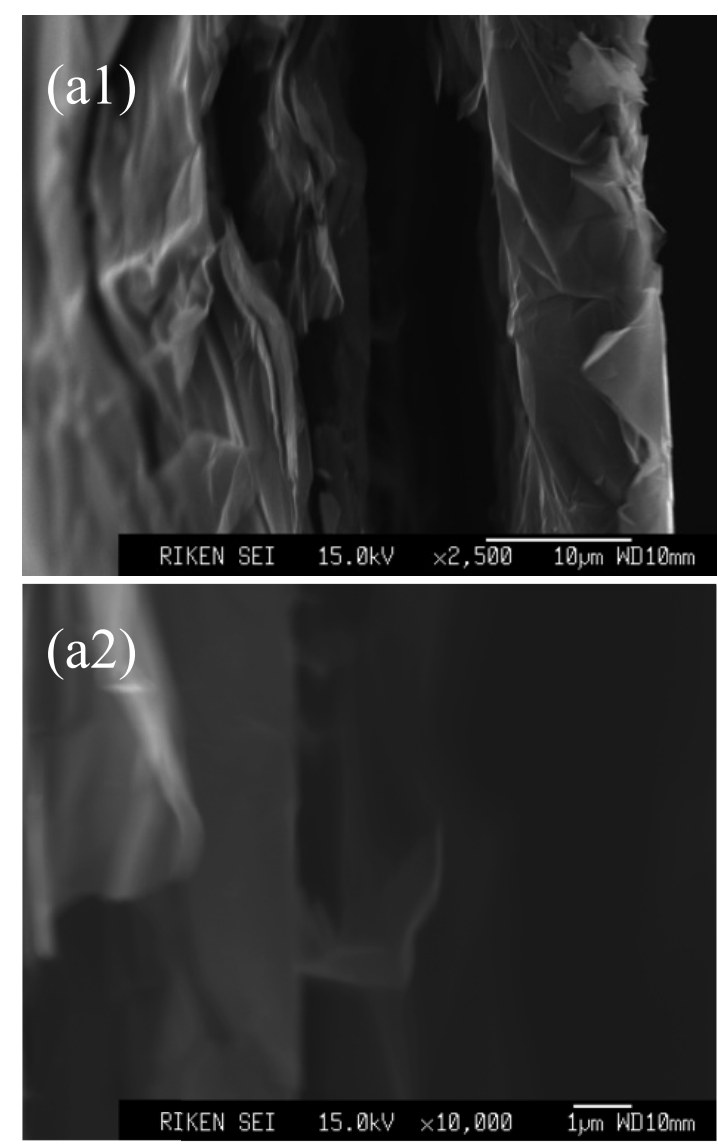

Fig. 4a. SEM images of the GS cross-section. (a1) and (a2): no-irradiated part.

\section{Summary}

We have been using KANEKA GSs as the second stripper for uranium acceleration since 2015. This GS stripper works successfully and provides stable high-intensity beams for a long period. Currently, the used GS disk is replaced after one beam time operation due to the increase in beam intensity. Used GSs seem to have no beamirradiation damage when observed visually. However, when observed using a SEM, damage of micrometerorder is observed on the surface and inside the GS. The reason for the damage may be due to weakening of the bond strength or lattice defects caused by the beam irradiation. It is noticed that the thickness of the GS is not changed by beam irradiation at the present intensity. Further analysis by Raman spectroscopy and TEM may resolve details. It is concluded that KANEKA's multilayer GS is a suitable material for use as charge strippers.
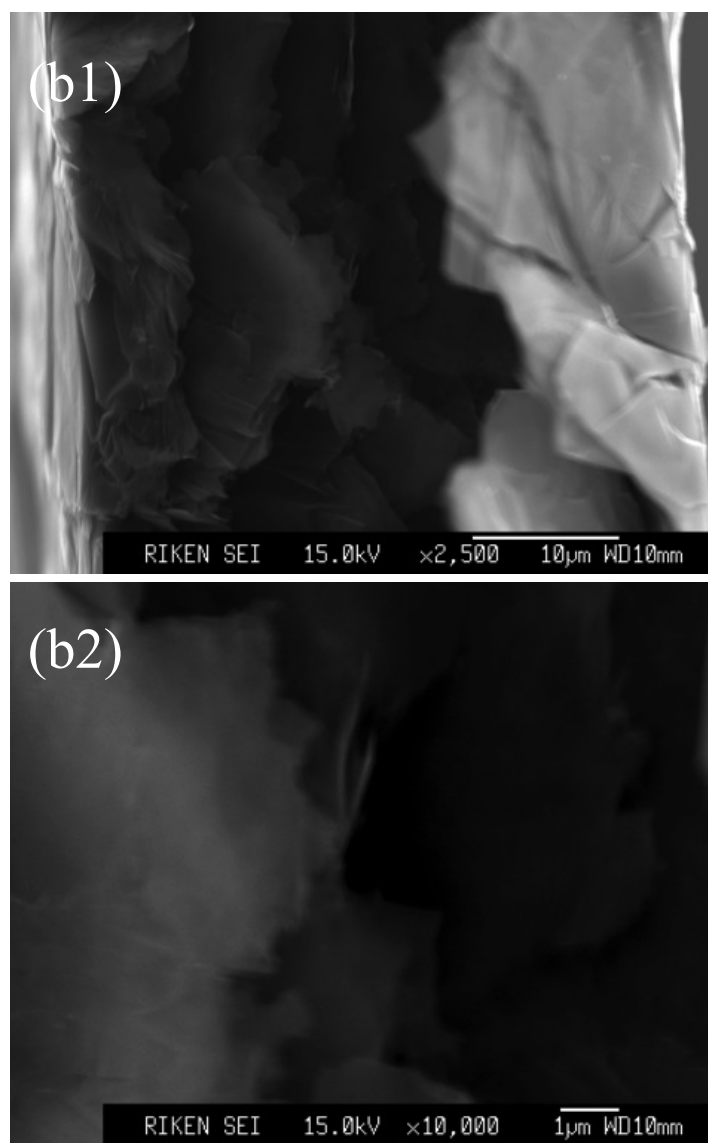

Fig. 4b. SEM images of the GS cross-section (b1) and (b2): beam-irradiated part

\section{References}

1. ACF-Metals Arizona Carbon Foil Co. Inc., URL: http://www.techexpo.com/firms/acf-metl.html

2. H. Hasebe, H. Okuno, H. Kuboki, H. Imao, N. Fukunishi, M. Kase, O. Kamigaito, AIP Conference Proceedings 1962, 030004 (2018)

3. A. Tatami, M. Tachibana, T. Yagi, M. Murakami, AIP Conference Proceedings 1962, 030005 (2018) 